\title{
Machine's Fault Diagnosis System Using Neural Networks
}

\author{
Toshiyuki ASAKURA, $\bigcirc$ Takashi KOBAYASHI, Shoji HAYASHI \\ Dept. of Mech. Eng., Fukui University, 3-9-1 Bunkyo, Fukui, 910-8507 Japan \\ Tel: +81-776-23-0500(x2764), Fax: +81-776-27-8748 \\ e-mail : asakura@scmc.mech.fukui-u.ac.jp
}

\begin{abstract}
This research develops a method of machine fault diagnosis system using neural networks and spectral analysis. Generally, it is difficult to diagnose the fault of a machine by the conventional mathematical method. In this research, normal and fault spectral data, which are obtained from operating machine, are learned by the diagnosis neural network. Next, when a fault occurs in the machine, the fault is detected using this fault diagnosis system. This diagnosis system can diagnose not only a known fault that was learned but also an unknown fault. And, it can also find the unknown fault pattern based on the known fault pattern. This diagnosis system diagnoses the fault based on only the change of sounds or behaviors obtained from a operating machine. Therefore, the characteristic of this method is that, as neural networks can identify machine system, a mathematical model is not need.
\end{abstract}

\section{Introduction}

Among many problems in industries, the most important factor is for each machine system to work in the normal state. In order to maintain a normal condition of a machine system, a fault prediction and diagnosis system are necessary, especially for those such as electrical generators, which run continuously for long time. The failures should be detected as soon as possible when failures occur, because if these machines run at an abnormal condition continuously, it may result in heavy loss and even loss of human lives. Up to date, though many kinds of diagnosis methods[1] have been developed, most of them have been based on traditional method of establishing mathematical model[2], analyzing variety of parameters and judging the operating condition of machine system. But, because of the complication of machine system, the uncertainty of operating condition and many nonlinear factors, it is in most cases very difficult, to establish mathematical model of machine structure and to know the operating conditions of the machine. Also, it is in some cases even impossible, to detect failures occurred when it is running. So that, many researchers are recently attracted in untraditional approaches[3].

This study develops a method of fault diagnosis based on machine fault diagnosis system using neural networks. In this method, the diagnosis system has the improved neural network. The diagnosis neural network learns the already obtained characteristics of normal and abnormal operating condition of machine system. The diagnosis system is constructed using the neural network that has finished learning. This diagnosis system diagnoses the fault and detects the fault pattern based on the known condition that it learned. If the unknown fault pattern occurred in the object machine, the diagnosis system can detect the unknown fault and can find the unknown fault pattern by the improved diagnosis neural network. Therefore, the proposed diagnosis system can diagnose not only the known fault pattern but also the unknown fault pattern. In this paper, through 


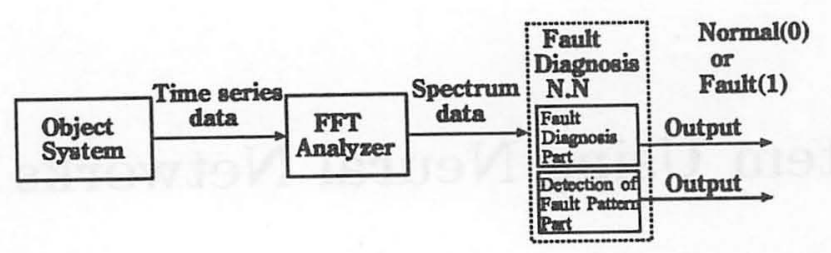

Fig. 1 Structure of fault diagnosis system

simulations, the effectiveness of proposed diagnosis system is verified.

\section{Fault diagnosis system}

\subsection{Structure of fault diagnosis system}

The structure of a fault diagnosis system is shown in Fig.1. This system is composed of FFT analyzer and the diagnosis neural network(NN). The output of NN is nearly 0 , if the object system is in the normal state. Otherwise, the output nearly 1. Using FFT analyzer, the spectral analysis is performed for time series data such as sounds or vibrations obtained from the object system.

The spectrum can be calculated by Eqs.(1) and (2) through FFT analyzer. In Eq.(1), $f(n)$ is time series data obtained from the object system, and $F(k)$ are frequency spectrum. In Eq.(2), $F_{R}(k)$ is a real part and $F_{I}(k)$ a imaginary part of frequency spectrum .

$$
\begin{aligned}
& F(k)=\sum_{n=0}^{N-1} f(n) e^{-j 2 \pi k n / N} \\
& P(k)=|F(k)|=F_{R}(k)^{2}+F_{I}(k)^{2}
\end{aligned}
$$

Where, $N$ is the number of sampling point, $\Delta t$ sampling time, $\Delta t$ sampling cycle, and $\Delta \omega$ frequency resolution. The following relation holds,

$$
\begin{aligned}
T & =1.0 / \Delta \omega \\
\Delta t & =T / N
\end{aligned}
$$

In this simulation, the sampling point $N$ is 1024 , and frequency resolution is $0.1[\mathrm{~Hz}]$, sampling cycle $10[s]$, and sampling time $9.8 \times 10^{-3}[s]$. The first input to NN is performed, when the first sampling cycle finished. After that, the power spectrum is recalculated each one sampling, and that data is inputted to NN each one sampling.

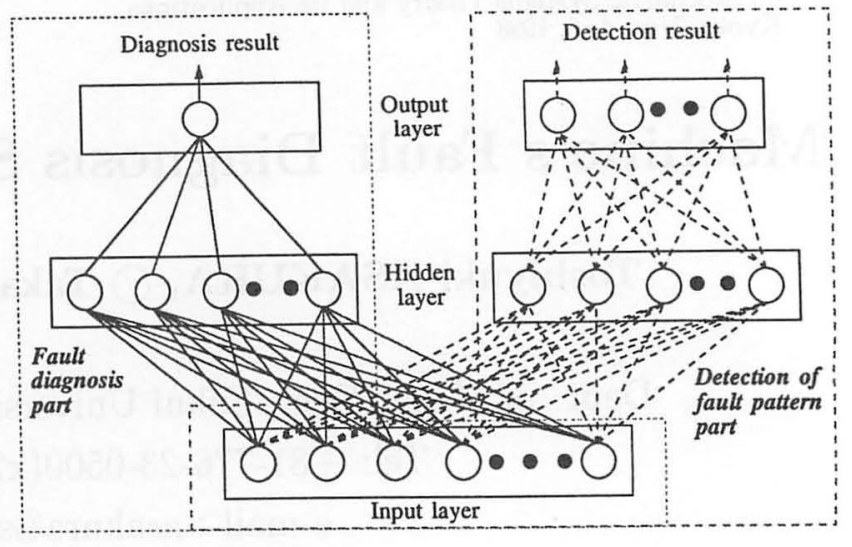

Fig. 2 Structure of diagnosis neural network

\subsection{Diagnosis neural network}

Generally, the ordinary diagnosis network has the sigmoid function for unit function. The diagnosis network learns both normal and fault data of the object system. Then, the diagnosis network diagnoses the fault and detects the fault pattern based on learned data. However, the diagnosis network often makes a wrong diagnosis to the unknown fault pattern. Therefore, the precision of fault diagnosis was not enough.

\subsubsection{Structure of diagnosis network}

The structure of NN is shown in Fig.2. The NN is hierarchical network and it composes fault diagnosis part and detection of fault pattern part. Each part of NN has input layer, hidden layer and output layer. And the input layer is common each part. The output layer of the diagnosis part has one unit to diagnose the fault. And, the number of the output layer unit of detection part is the same the number of detected fault patterns. Each output layer unit of detection part detects only allocated fault pattern. The number of other layer unit is changeable correspond to the object system and it is decided trial and error. The input data to $\mathrm{NN}$ is power spectrum obtained through FFT analyzer, and the range of frequency of power spectrum is changeable correspond to the object system. The fault diagnosis part judges only whether the object system is a normal or an abnormal condition. If fault occurs, the fault pattern detection is performed by detection of fault pattern part. 


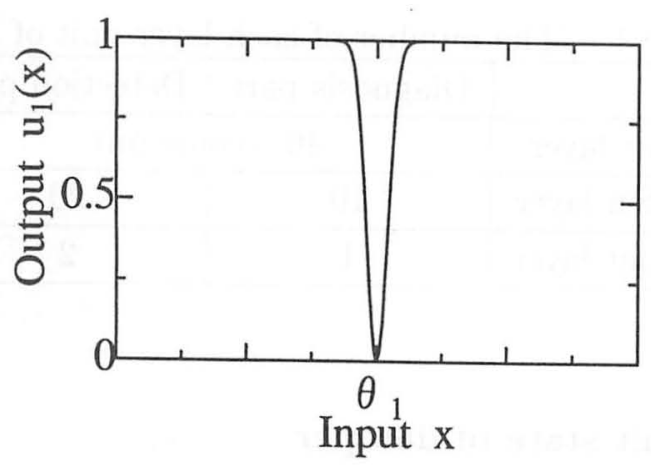

(a) Output pattern of Eq.(5)

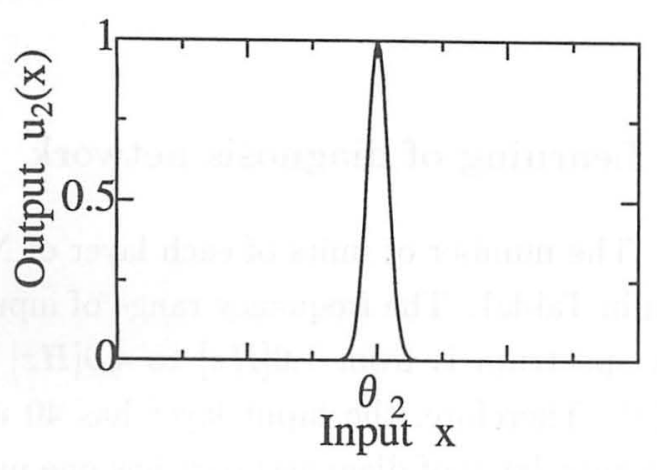

(b) Output pattern of Eq.(6)

Fig. 3 Output pattern of Output functions

\subsubsection{Output layer function}

NN which has the output function such as shown in Fig. 3 proposed to improve the precision of fault diagnosis. The output functions are given by Eqs.(5) and (6), and their output patterns are shown in Fig.3.

$$
\begin{aligned}
& u_{1}(x)=\frac{-A_{1}}{\sqrt{2 \pi \sigma_{1}}} e^{-\left(x-\theta_{1}\right)^{2} / 2 \sigma_{1}{ }^{2}}+1.0 \\
& u_{2}(x)=\frac{A_{2}}{\sqrt{2 \pi \sigma_{2}}} e^{-\left(x-\theta_{2}\right)^{2} / 2 \sigma_{2}{ }^{2}}
\end{aligned}
$$

In Eq.(5), $A_{1}$ is a parameter to restrict output value of $\mathrm{NN}$ from 0 to $1, \sigma_{1}$ a parameter to restrict normal condition, and $\theta_{1}$ a threshold of the unit. In Eq.(6), $A_{2}$ is a parameter to restrict output value of $\mathrm{NN}$ from 0 to $1, \sigma_{2}$ a parameter to restrict detected fault pattern, and $\theta_{2}$ a threshold of the unit. The Eq.(5) is used for fault diagnosis part and the Eq.(6) used for detection of the fault pattern part. The Eq.(5) will be output 0 only for normal state by restricting the range of input $\mathrm{x}$ of

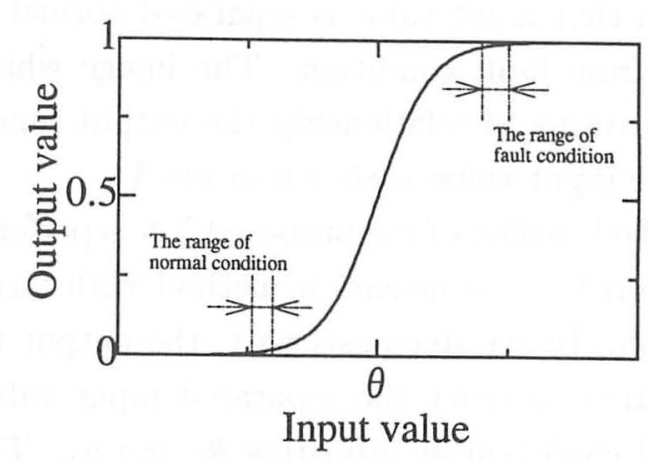

Fig. 4 Learning image of sigmoid function

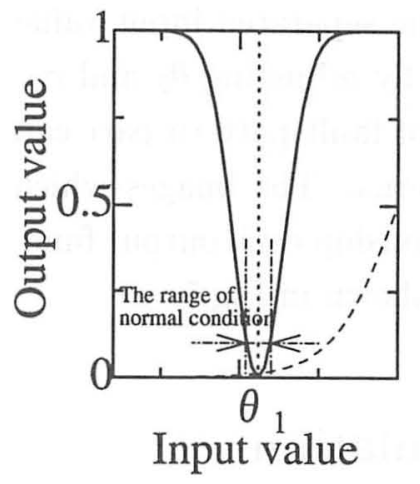

(a) Diagnosis part

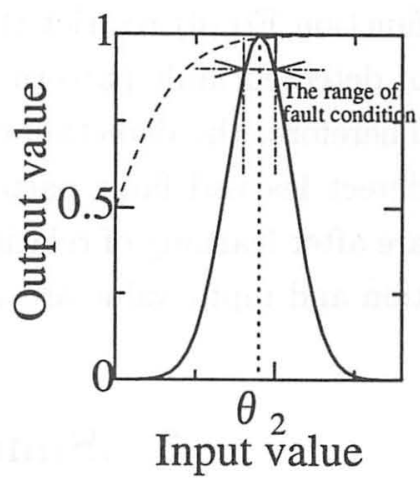

(b) Detection part
Fig. 5 Learning image of proposed function

normal state at learning process. When the input $\mathrm{x}$, which is different from normal state, was added, the diagnosis network output 1 . Therefore, the diagnosis network can diagnose not only the known fault but also the unknown that. The Eq.(6) will be output 1 for learned fault pattern by reacting the range of input $\mathrm{x}$ of learned fault pattern at learning process. The diagnosis network can also find the unknown fault pattern. Accordingly, the precision of both fault diagnosis and fault pattern detection will be better comparing with the ordinary diagnosis network.

\subsubsection{Leaning method of diagnosis network}

In general, the learning of ordinary neural network is performed by back propagation method. In this method, the connection weight and the threshold is adjusted to reduce the error of output value. And the learning is achieved by repeating this process. In the output layer, it can 
be seen that input value is separated normal condition from fault condition. The image which is after learning of relationship the output function and the input value is shown in Fig.4

The learning of the proposed $\mathrm{NN}$ is performed based on back propagation method with sigmoid function. In the diagnosis part, the output function Eq.(5) restrict the separated input value of normal condition by adjusting $\theta_{1}$ and $\sigma_{1}$. Therefore, the fault diagnosis part can diagnose whether normal or faults. In the detection part, the output function Eq.(6) restrict the separated input value of detected fault pattern by adjusting $\theta_{2}$ and $\sigma_{2}$. Therefore, the detection of fault pattern part can detect learned fault patterns. The images which are after learning of relationship each output function and input value are shown in Fig.5.

\section{Simulation}

\subsection{Simulation model}

As the simulation model of the diagnosis object, Duffing type nonlinear system is used. The dynamics of the system is described by

$$
m \ddot{x}+c \dot{x}+k x+\alpha x^{3}=q(t)+w(t)
$$

where, $m$ is the mass, $x$ the displacement of the object, $c$ a coefficient of viscosity, and $k x+\alpha x^{3}$ force of a spring. Furthermore, $q(t)$ is an external force which is given by $q(t)=Q \cos \omega t$, and $w(t)$ a noise added to the system (It is assumed that $w(t)$ is a white gaussian noise.).

In this study, the object system is set up to normal and abnormal state for spring and damper. The fault diagnosis is performed to spring and damper of the object system. The standard normal and abnormal state for both spring and damper are set as the following parameters.

$$
\begin{aligned}
& \text { " Normal state } \\
& m=1.0, k=2.0, \alpha=50.0, c=2.0, Q=1.0 \\
& \omega=2.0[\mathrm{rad} / \mathrm{s}], \dot{x}(0)=0.01, x(0)=0.01 \\
& \text { " Fault state of spring } \\
& m=1.0, k=1.0, \alpha=25.0, c=2.0, Q=1.0 \\
& \omega=2.0[\mathrm{rad} / \mathrm{s}], \dot{x}(0)=0.01, x(0)=0.01
\end{aligned}
$$

Table 1 The number of each layer unit of NN

\begin{tabular}{|c|c|c|}
\hline & Diagnosis part & Detection part \\
\hline Input layer & \multicolumn{2}{|c|}{40 (common) } \\
\hline Hidden layer & 10 & 20 \\
\hline Output layer & 1 & 2 \\
\hline
\end{tabular}

$$
\begin{aligned}
& \text { - Fault state of damper } \\
& m=1.0, k=2.0, \alpha=50.0, c=1.0, Q=1.0 \\
& \omega=2.0[\mathrm{rad} / \mathrm{s}], \dot{x}(0)=0.01, x(0)=0.01
\end{aligned}
$$

\subsection{Learning of diagnosis network}

The number of units of each layer of NN is shown in Table1. The frequency range of inputted power spectrum is from $1.0[\mathrm{~Hz}]$ to $4.9[\mathrm{~Hz}]$ each $0.1[H z]$. Therefore, the input layer has 40 units. The output layer of diagnosis part has one unit to diagnose whether normal or faults. And detection part has two output layer units to detect spring's fault and damper's fault. And, the number of each hidden layer unit is decided trial and error. The initial connection weights and thresholds are set up at random from -1.0 to 1.0. The output function's initial parameters of the diagnosis part are set up $A_{1}=1.7724$ and $\sigma_{1}=0.50$. The initial parameters of output function to detect the spring's fault are set up $A_{21}=1.7724$ and $\sigma_{21}=0.50$. The initial parameters of output function to detect the damper's fault are set up $A_{22}=1.7724$ and $\sigma_{22}=0.50$.

In the learning, it is assumed that the normal sate and two fault pattern of the diagnosis object have already known. The two fault patterns are spring's fault and damper's fault. Each state of the object is described in the previous part. The learning is performed using 12 data including noise (normal 4, spring's fault 4, and damper's fault 4). The learning of fault diagnosis part finishes at about 9600 learning step. Then, the rms error between output and teacher signal is 0.0097 . By the learning, the output function's parameters of diagnosis part are decided $A_{1}=0.250663$ and 


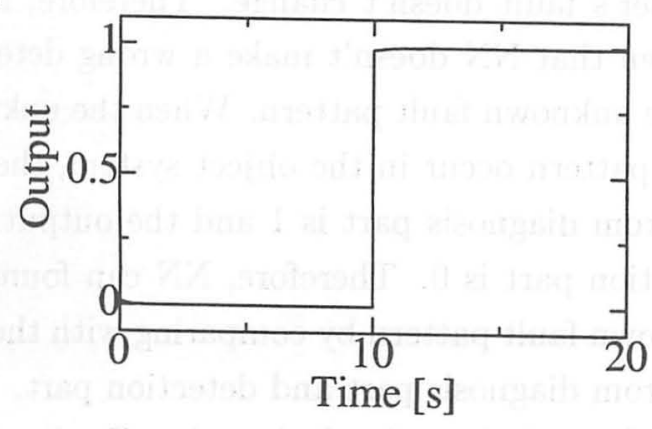

(a) Diagnosis result

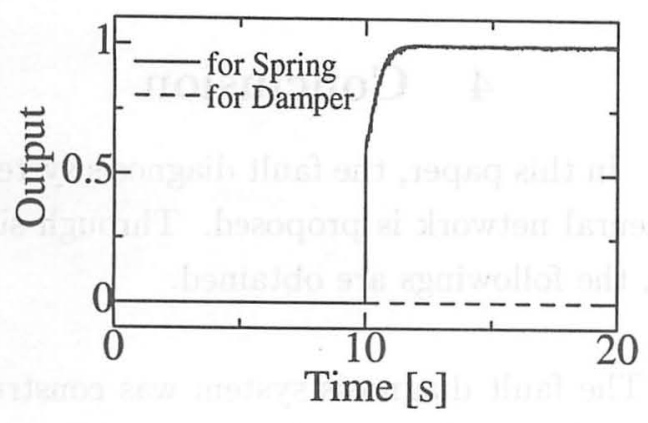

(b) Detection result

Fig. 6 Fault diagnosis and detection result

$\sigma_{1}=0.01$. The learning of fault pattern detection part finishes at about 3200 learning steps. Then, the rms error between output and teacher signal is 0.0081 . By the learning, the parameter of output function to detect the spring's fault decided $A_{21}=0.560499, \sigma_{21}=0.05$, and the parameter of output function to detect the damper's fault decided $A_{22}=0.560499, \sigma_{22}=0.05$.

The process of the fault diagnosis simulation is explained as follows. The first input to $\mathrm{NN}$ is performed, when the first sampling cycle finished. After that, the power spectrum is recalculated each one sampling, and that data is inputted to NN each one sampling. And the diagnosis result is judged by the each output from NN. The diagnosis and detection results for learned spring's fault are shown in Fig.6, in which Fig.6(a) is diagnosis result and Fig.6(b) detection result. In Fig.6, when the behavior changes from the normal state to abnormal state at $t=10 \mathrm{sec}$, the output of diagnosis part changes to 1 from 0 . And, the output to detect spring's fault changes to 1 from 0 . There-

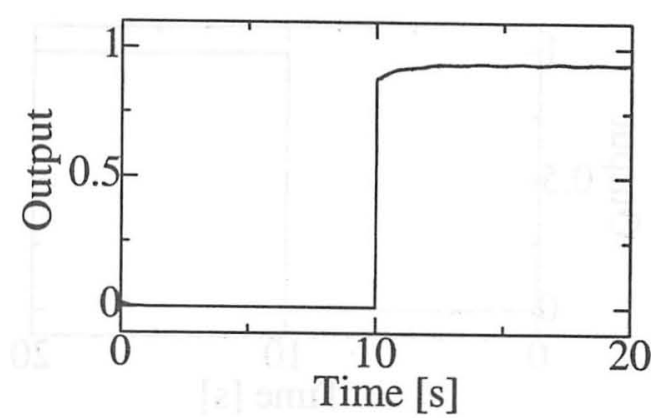

(a) Diagnosis result

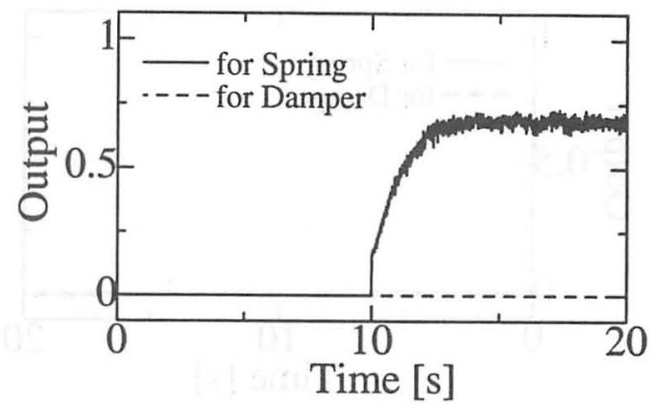

(b) Detection result

Fig. 7 Fault diagnosis and detection result

fore, it can be seen that NN diagnoses the fault, and it detect the fault pattern exactly. It is also confirmed that the damper's fault is diagnosed and detected by NN.

\subsection{Diagnosis for unknown condition}

In generally, the normal state of the object can be found from operating machine, but the abnormal state can't be found all. However, the diagnosis system must diagnose the unknown fault and it must not perform incorrect fault pattern detection to the unknown fault pattern.

In this section, the fault diagnosis is performed for two fault states which are different from learning fault state using after learning $\mathrm{NN}$ in previous part. The one fault state is the unlearned spring's fault $(k=1.3, \alpha=25.0)$ which is different from learned state. The another fault state is both spring and damper fault $(k=1.0, \alpha=25.0, c=$ 1.0) which is the unknown fault pattern.

The diagnosis result for unlearned spring's 


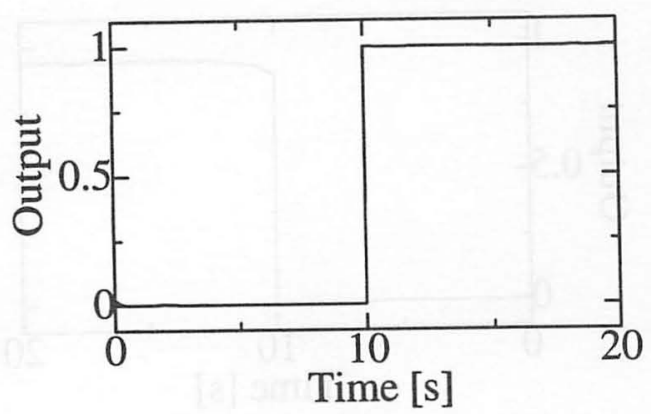

(a) Diagnosis result

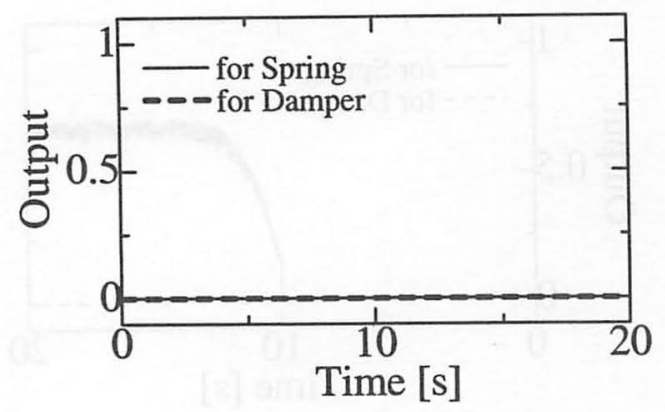

(b) Detection result

Fig. 8 Fault diagnosis and detection result

fault is shown in Fig.7, in which Fig.7(a) is diagnosis result and Fig.7(b) detection result. In Fig.7, when the behavior changes from the normal state to the unlearned abnormal state at $t=10 \mathrm{sec}$, the output of diagnosis part changes to nearly 1 from 0 . Therefore, it can be seen that NN can also diagnose the unlearned fault state exactly. And, the output of to detect spring's fault changes to nearly 0.7 from 0 . Therefore, it can be seen that NN also detects the fault pattern exactly for unlearned fault state of the spring. It is also confirmed that the unlearned damper's fault is diagnosed and detected by NN.

The diagnosis result for unknown fault pattern is shown in Fig.8, in which Fig.8(a) is diagnosis result and Fig.8(b) detection result. In Fig.8, when the behavior changes from the normal state to the unknown abnormal pattern at $t=10$ sec, the output of diagnosis part changes to 1 from 0 . Therefore, it can be seen that NN can also diagnose the unknown fault pattern exactly. And, the each output to detect spring's fault and damper's fault doesn't change. Therefore, it can be seen that NN doesn't make a wrong detection to the unknown fault pattern. When the unknown fault pattern occur in the object system, the output from diagnosis part is 1 and the output from detection part is 0 . Therefore, $\mathrm{NN}$ can found the unknown fault pattern by comparing with the output from diagnosis part and detection part.

Through these simulation, the effectiveness of proposed diagnosis system is verified.

\section{Conclusion}

In this paper, the fault diagnosis system using neural network is proposed. Through simulations, the followings are obtained.

1. The fault diagnosis system was constructed, which can diagnose the fault and can detect the fault pattern using spectrum data of the object system.

2. This fault diagnosis system can diagnose not only the learned fault but also the unlearned fault.

3. This fault diagnosis system can find unknown fault pattern when that is occur in the object system.

\section{Reference}

[1] M.MPolycarpou, "Learning Methodology for Failure Detection and Accommodation", IEEE Contr. Sys., June 1995

[2] D.Nguyen and B.Windrow, "Neural networks for selflearning control system", IEEE Control System Magazine, Vol.10,No.3,1990

[3] T.Asakura, Baojie Xu, Shoji Hayashi and Takashi Kobayashi, "Machine Fault Diagnosis System Using Neural Networks", Proc. of 28th ISCIE International Symposium on Stochastic Theory and Its Application, Kyoto, (1996)

[4] D.E.Rumelhart, G.E Hinton and R.J.Williams, "Learning Representations by Back-propagation Errors", Nature, Vol.323, No.9, (1986),533

[5] H.T. Sheu and Y.H. Chang,"Robust Fault Diagnosis for Large-Scale Analog Circuits with Mesurement Noises",IEEE, TRANS., Vol.44 No.3 March 1997

[6] T.Asakura, T.Kobayashi and S.Hayashi,"A study of Fault Diagnosis System Using Neural Networks", Proc. of 29th ISCIE International Symposium on Stochastic Theory and Its Application, Tokyo, pp.19-24(1997) 